Bull. Korean Math. Soc. 51 (2014), No. 2, pp. 401-408

http://dx.doi.org/10.4134/BKMS.2014.51.2.401

\title{
ON THE MULTIPLE HURWITZ-EULER ETA FUNCTIONS AND THE SUMS OF PRODUCTS OF THE EULER NUMBERS
}

\author{
JIN-WoO SON
}

\begin{abstract}
We establish a new approach for the sums of products of the Euler numbers by using the relation of values at non-positive integers of the representation of the multiple Hurwitz-Euler eta function in terms of the Hurwitz-Euler eta function.
\end{abstract}

\section{Introduction}

The Euler polynomials can be defined by means of the following generating function (see, for details, [1, p. 519]; see also [7, 9] and the references cited in each of these recent investigations on the subject):

$$
\left(\frac{2}{e^{t}+1}\right) e^{x t}=\sum_{n=0}^{\infty} E_{n}(x) \frac{t^{n}}{n !} .
$$

When $x=0$ in (1.1) $E_{n}(0)=E_{n}$ are called the $n$th Euler numbers. This numbers $E_{n}$ appear in many instances in pure and applied mathematics, most notably in number theory, finite differences calculations, and asymptotic analysis. Therefore, the efficient computation for the sums of products of the numbers $E_{n}$ is of great interest.

A well-known relation among the numbers $E_{k}$ is

$$
\begin{aligned}
\sum_{i=0}^{k}\left(\begin{array}{l}
k \\
i
\end{array}\right) E_{i} E_{k-i} & =(-1)^{k+1} 2\left(E_{k}-E_{k+1}\right) \\
& =2\left(E_{k}+E_{k+1}\right) \quad(k>0),
\end{aligned}
$$

the second equality follows from $E_{2 i}=0$ with $i \in \mathbb{N}$. This has been found by many authors, including Euler (see $[2,3,4,6]$ ). In [2], Dilcher remarked that: "It may be of interest to find formulas of the above type for sums of products of generalized Bernoulli numbers."

Received July 18, 2012; Revised May 27, 2013.

2010 Mathematics Subject Classification. 11B68.

Key words and phrases. Euler numbers, Hurwitz-Euler eta function, multiple HurwitzEuler eta function, sums of products. 
Many results on sums of products of analogues of Bernoulli numbers are also known. For example, sums of products of Bernoulli numbers [2, 4] and sums of products of Apostol-Bernoulli numbers [5] were studied. Also, some similar formulas for (1.2) on the Bernoulli numbers have been investigated by in [6].

The generalized Euler polynomials, $E_{n}^{(m)}(x)$, are defined by the generating functions:

$$
\left(\frac{2}{e^{t}+1}\right)^{m} e^{x t}=\sum_{n=0}^{\infty} E_{n}^{(m)}(x) \frac{t^{n}}{n !} .
$$

Clearly, we find from (1.3) that $E_{n}^{(1)}(x)=E_{n}(x)$ and $E_{n}^{(1)}=E_{n}$. In particular, $E_{n}^{(k)}(0)=E_{n}^{(k)}$ are the generalized Euler numbers (see [1, Definition 3]).

The alternating Hurwitz zeta function (or, equivalently, Hurwitz-Euler eta function) $\eta(s)$ is defined by (see $[1$, p. 514])

$$
\eta(s, x)=\sum_{n=0}^{\infty} \frac{(-1)^{n}}{(n+x)^{s}} \quad(\Re(s)>0 ; x>0) .
$$

The special cases of (1.4) when $x=1$, denote by $\eta(s, 1):=\eta(s)=\sum_{n=1}^{\infty} \frac{(-1)^{n-1}}{n^{s}}$, where $\Re(s)>0$, is called the Dirichlet eta function (or the alternating Riemann zeta function).

Let $x$ be a positive real number. The multiple Hurwitz-Euler eta function $\eta_{m}(s, x)$ is defined by

$$
\eta_{m}(s, x)=\sum_{n_{1}, \ldots, n_{m}=0}^{\infty} \frac{(-1)^{n_{1}+\cdots+n_{m}}}{\left(x+n_{1}+\cdots+n_{m}\right)^{s}},
$$

where $\Re(s)>0$ and $m \in \mathbb{N}$. This can be continued analytically in the whole $s$-plane. For further results concerning these classes of multiple Hurwitz-Euler eta function $\eta_{m}(s, x)$, we refer to the recent works by Choi and Srivastava [1, $\S 3]$.

It is easy to see that

$$
\eta_{1}(s, x)=\eta(s, x) .
$$

In the present paper, by using the relation of values at non-positive integers of the representation of the multiple Hurwitz-Euler eta function in terms of the Hurwitz-Euler eta function, we prove a new approach for the sums of products of the Euler numbers.

Before stating of the main result, we introduce the following notations

$$
(x)_{m}=x(x+1) \cdots(x+m-1)=\sum_{j=0}^{m}\left[\begin{array}{c}
m \\
j
\end{array}\right] x^{j},
$$

where $\left[\begin{array}{c}m \\ j\end{array}\right]$ are the Stirling cycle numbers, defined recursively by

$$
\left[\begin{array}{c}
m \\
j
\end{array}\right]=(m-1)\left[\begin{array}{c}
m-1 \\
j
\end{array}\right]+\left[\begin{array}{c}
m-1 \\
j-1
\end{array}\right], \quad\left[\begin{array}{c}
m \\
0
\end{array}\right]= \begin{cases}1, & m=0 \\
0, & m \neq 0\end{cases}
$$


(see $[1,4,5,8])$. Then

$$
\left(\begin{array}{c}
n+m-1 \\
m-1
\end{array}\right)=\sum_{l=0}^{m-1} p_{m, l}(x)(x+n)^{l},
$$

where $p_{m, l}(x)$ is a polynomial in $x$ defined by

$$
p_{m, l}(x)=\frac{1}{(m-1) !} \sum_{j=l}^{m-1}(-x)^{j-l}\left(\begin{array}{l}
j \\
l
\end{array}\right)\left[\begin{array}{c}
m \\
j+1
\end{array}\right]
$$

(cf. $[1,4,5,8])$.

If we let $n=y-x,(1.9)$ may be rewritten as

$$
\left(\begin{array}{c}
y-x+m-1 \\
m-1
\end{array}\right)=\sum_{l=0}^{m-1} p_{m, l}(x) y^{l} .
$$

In (1.11), replace $x$ by $m$, we find easily that

$$
p_{m, l}(m)=\frac{s(m, l+1)}{(m-1) !},
$$

where $s(m, l+1)$ denotes the Stirling numbers of the first kind (see [1, (1.18)]).

By applying (1.11) and (1.12), we obtain Lemma 1.1 below.

Lemma 1.1. Let $m \in \mathbb{N}$. Then we have

$$
\sum_{l=0}^{m-1} s(m, l+1) i^{l}=0 \quad(1 \leq i \leq m-1) .
$$

Now we state our main result.

Theorem 1.2. Let $n \in \mathbb{N}$. Then we have

$$
\sum_{\substack{n_{1}+\cdots+n_{m}=n \\
n_{1}, \ldots, n_{m} \geq 0}}\left(\begin{array}{c}
n \\
n_{1}, \ldots, n_{m}
\end{array}\right) E_{n_{1}} \cdots E_{n_{m}}=\frac{(-1)^{m+n}}{(m-1) !} 2^{m-1} \sum_{l=0}^{m-1} s(m, l+1) E_{n+l},
$$

where $\left(\begin{array}{c}n \\ n_{1}, \ldots, n_{m}\end{array}\right)$ are multinomial coefficients defined by

$$
\left(\begin{array}{c}
n \\
n_{1}, \ldots, n_{m}
\end{array}\right)=\frac{n !}{n_{1} ! \cdots n_{m} !} .
$$

Remark 1.3. This is a generalization of (1.2).

\section{Main results}

By slightly modifying the method described in [1], we obtain the following result which connects the special values of multiple Hurwitz-Euler eta function $\eta_{m}(s, x)$ to generalized Euler polynomials $E_{n}^{(m)}(x)$, which is also well-known (see for example [1, Corollary 3$]$ ). 
Lemma 2.1. For $\ell \in \mathbb{N}_{0}$, we have

$$
\eta_{m}(-\ell, x)=\frac{1}{2^{m}} E_{\ell}^{(m)}(x) .
$$

Proof. The multiple Hurwitz-Euler eta function $\eta_{m}(s, x)$ is expressed as an integral,

$$
\Gamma(s) \eta_{m}(s, x)=\int_{0}^{\infty} \frac{e^{-x t} t^{s-1}}{\left(1+e^{-t}\right)^{m}} d t \quad(\Re(s)>0 ; m \in \mathbb{N}),
$$

where $\Gamma(s)=\int_{0}^{\infty} t^{s-1} e^{-t} d t$ (see [1, Theorem 3]). By slightly modifying the method described in [1, Theorem 4], we can extend the above integral representation to all complex $s=\sigma+i t$ with $\sigma>0$. Indeed, let $C$ denote the contour which starts from $+\infty$, runs on the real axis, encircling the origin once counter-clockwise on the circle of small radius $\epsilon$ with the center at 0 , runs again the real axis and returns to $+\infty$. Since

$$
\int_{C} \frac{e^{-x z} z^{s-1}}{\left(1+e^{-z}\right)^{m}} d z=\left(e^{2 \pi i s}-1\right) \int_{0}^{\infty} \frac{e^{-x t} t^{s-1}}{\left(1+e^{-t}\right)^{m}} d t
$$

we have $(\operatorname{as} \Gamma(s) \Gamma(1-s)=\pi / \sin (\pi s)(s \in \mathbb{C} \backslash \mathbb{Z}))$

$$
\eta_{m}(s, x)=\frac{e^{-\pi i s} \Gamma(1-s)}{2 \pi i} \int_{C} \frac{e^{-x z} z^{s-1}}{\left(1+e^{-z}\right)^{m}} d z .
$$

This is the main virtue to obtain a contour integral representation for an analytic function. In particular, we see that $\eta_{m}(s, x)$ can be continued analytically to the whole $s$-plane. Put

$$
g^{(m)}(x, t)=\left(\frac{2}{e^{t}+1}\right)^{m} e^{x t}
$$

Thus, if we divide the infinite integral into two parts

$$
\begin{aligned}
& 2^{-m} \int_{0}^{\infty} t^{s-1} g^{(m)}(x,-t) d t \\
= & 2^{-m}\left(\int_{0}^{1} t^{s-1} g^{(m)}(x,-t) d t+\int_{1}^{\infty} t^{s-1} g^{(m)}(x,-t) d t\right),
\end{aligned}
$$

it is easily seen that the second term is an entire function of $s$. Using the Taylor expansion

$$
g^{(m)}(x,-t)=\sum_{n=0}^{N}(-1)^{n} E_{n}^{(m)}(x) \frac{t^{n}}{n !}+R_{N}^{(m)}(x,-t),
$$


where $R_{N}^{(m)}(x,-t)=\sum_{n=N+1}^{\infty}(-1)^{n} E_{n}^{(m)}(x) \frac{t^{n}}{n !}$, we obtain

$$
\begin{aligned}
\int_{0}^{1} t^{s-1} g^{(m)}(x,-t) d t & =\sum_{n=0}^{N}(-1)^{n} \frac{E_{n}^{(m)}(x)}{n !} \int_{0}^{1} t^{s-1+n} d t+H_{N}(s) \\
& =\sum_{n=0}^{N} \frac{E_{n}^{(m)}(x)}{n !} \frac{(-1)^{n}}{s+n}+H_{N}(s),
\end{aligned}
$$

where $H_{N}(s)$ is holomorphic for $\Re(s)>0$. Therefore sufficiently large $N$ we have

$$
\begin{aligned}
2^{m} \eta_{m}(s, x)= & \sum_{n=0}^{N} \frac{E_{n}^{(m)}(x)}{n ! \Gamma(s)} \frac{(-1)^{n}}{s+n}+\frac{1}{\Gamma(s)} H_{N}(s) \\
& +\frac{1}{\Gamma(s)} \int_{1}^{\infty} t^{s-1} g^{(m)}(x,-t) d t
\end{aligned}
$$

For $\ell \in \mathbb{N}_{0}$, we have

$$
2^{m} \lim _{s \rightarrow-\ell}(s+\ell) \Gamma(s) \eta_{m}(s, x)=E_{\ell}^{(m)}(x) \frac{(-1)^{\ell}}{\ell !} .
$$

If $\ell \in \mathbb{N}_{0}$, we have $\lim _{s \rightarrow-\ell}(s+\ell) \Gamma(s)=(-1)^{\ell} / \ell$ ! and thus we obtain

$$
\eta_{m}(-\ell, x)=2^{-m} E_{\ell}^{(m)}(x)
$$

which completes the proof of the lemma.

Lemma 2.2. If $n \in \mathbb{N}_{0}$, then

$$
E_{n}^{(m)}=\sum_{n_{1}+\cdots+n_{m}=n}\left(\begin{array}{c}
n \\
n_{1}, \ldots, n_{m}
\end{array}\right) E_{n_{1}} \cdots E_{n_{m}},
$$

where the sum is over all nonnegative integers $n_{1}, \ldots, n_{m}$ with $n_{1}+\cdots+n_{m}=n$.

Lemma 2.3. Let $m$ be a positive integer. Then

$$
\eta_{m}(s, x)=\sum_{l=0}^{m-1} p_{m, l}(x) \eta(s-l, x) .
$$

Proof. The number of solutions of $n_{1}+\cdots+n_{m}=n\left(n \in \mathbb{N}_{0},\left(n_{1}, \ldots, n_{m}\right) \in\right.$ $\mathbb{N}_{0}^{m}$ ) is equal to the coefficient of $x^{n}$ in the expansion of the Maclaurin series of $(1-x)^{-m}$, that is, $\left(\begin{array}{c}-m \\ n\end{array}\right)$. Hence the multiple Hurwitz-Euler eta function $\eta_{m}(s, x)$ may be rewritten as follows:

$$
\eta_{m}(s, x)=\sum_{n=0}^{\infty}\left(\begin{array}{c}
m+n-1 \\
m-1
\end{array}\right) \frac{(-1)^{n}}{(x+n)^{s}} .
$$

This can be continued analytically in the whole $s$-plane (for details see $[1,8]$ ). Therefore, by (1.9) and (2.1), we have the desired result. 


\section{Proof of Theorem 1.2}

It is easy to see that

$$
\frac{2 e^{(x+1) t}}{e^{t}+1}+\frac{2 e^{x t}}{e^{t}+1}=2 e^{x t}
$$

From (1.1) and (3.1), we have

$$
\sum_{n=0}^{\infty}\left(E_{n}(x+1)+E_{n}(x)\right) \frac{t^{n}}{n !}=\sum_{n=0}^{\infty} 2 x^{n} \frac{t^{n}}{n !} .
$$

Equating the coefficients of $t^{n}$ in this identity, we obtain

$$
E_{n}(x+1)+E_{n}(x)=2 x^{n} \quad\left(n \in \mathbb{N}_{0}\right) .
$$

Letting $x=0$ in the above identity, we have

$$
E_{n}(1)+E_{n}=2 \delta_{0, n},
$$

where $\delta_{i, j}$ is the Kronecker symbol defined by $\delta_{i, i}=1$ and $\delta_{i, j}=0$ for $i \neq j$. Substituting $x=1, \ldots, m-1$ in (3.3), alternating adding and subtracting the results, we obtain

$$
(-1)^{m-1} E_{n}(m)+E_{n}=2 \sum_{i=0}^{m-1}(-1)^{i} i^{n} \quad(m, n \in \mathbb{N}) .
$$

Assuming $m \in \mathbb{N}$. Then using (1.3), we get

$$
(-1)^{n} E_{n}^{(m)}(m-x)=E_{n}^{(m)}(x) .
$$

From Lemma 2.1 and (3.6), we obtain

$$
\begin{aligned}
\eta_{m}(-n, x) & =\frac{1}{2^{m}} E_{n}^{(m)}(x) \\
& =\frac{(-1)^{n}}{2^{m}} E_{n}^{(m)}(m-x) .
\end{aligned}
$$

By (1.6) and setting $m=1$ in Lemma 2.1 , we obtain

$$
\eta(-n, x)=\frac{1}{2} E_{n}(x) .
$$

Replacing $x$ by $m$ in (3.7), using Lemma 2.3, (1.12), (3.5) and (3.8), the left side of (3.7) can be written as

$$
\begin{aligned}
\eta_{m}(-n, m) & =\sum_{l=0}^{m-1} \frac{s(m, l+1)}{(m-1) !} \eta(-n-l, m) \\
& =\frac{1}{2} \sum_{l=0}^{m-1} \frac{s(m, l+1)}{(m-1) !} E_{n+l}(m) \\
& =(-1)^{m} \frac{1}{2} \sum_{l=0}^{m-1} \frac{s(m, l+1)}{(m-1) !}\left(E_{n+l}-2 \sum_{i=0}^{m-1}(-1)^{i} i^{n+l}\right) .
\end{aligned}
$$


From Lemma 1.1, we have

$(3.10)$

$$
\sum_{l=0}^{m-1} s(m, l+1) \sum_{i=0}^{m-1}(-1)^{i} i^{n+l}=\sum_{i=0}^{m-1}(-1)^{i} i^{n}\left(\sum_{l=0}^{m-1} s(m, l+1) i^{l}\right)=0(n \in \mathbb{N}) .
$$

Now, we set $n \in \mathbb{N}$. From (3.9) and (3.10), we have

$$
\eta_{m}(-n, m)=(-1)^{m} \frac{1}{2} \sum_{l=0}^{m-1} \frac{s(m, l+1)}{(m-1) !} E_{n+l} .
$$

On the other hand, from Lemma 2.1 and (3.6), we have the following

$$
\eta_{m}(-n, m)=\frac{1}{2^{m}} E_{n}^{(m)}(m)=\frac{(-1)^{n}}{2^{m}} E_{n}^{(m)} .
$$

Using Lemma 2.2 and (3.12), it is easily seen that

$$
\eta_{m}(-n, m)=\frac{(-1)^{n}}{2^{m}} \sum_{n_{1}+\cdots+n_{m}=n}\left(\begin{array}{c}
n \\
n_{1}, \ldots, n_{m}
\end{array}\right) E_{n_{1}} \cdots E_{n_{m}} .
$$

Thus, in view of (3.11) and (3.13), we find that

$$
\sum_{n_{1}+\cdots+n_{m}=n}\left(\begin{array}{c}
n \\
n_{1}, \ldots, n_{m}
\end{array}\right) E_{n_{1}} \cdots E_{n_{m}}=(-1)^{m+n} 2^{m-1} \sum_{l=0}^{m-1} \frac{s(m, l+1)}{(m-1) !} E_{n+l},
$$

where the sum is over all nonnegative integers $n_{1}, \ldots, n_{m}$ with $n_{1}+\cdots+n_{m}=n$. This completes the proof of our theorem.

Remark 3.1. Note that the sums of products of the Genocchi numbers are derivable as corollaries of the results presented herein.

Acknowledgements. This work was supported by a Kyungnam University Foundation grant in 2011.

\section{References}

[1] J. Choi and H. M. Srivastava, The multiple Hurwitz zeta function and the multiple Hurwitz-Euler eta function, Taiwanese J. Math. 15 (2011), no. 2, 501-522.

[2] K. Dilcher, Sums of products of Bernoulli numbers, J. Number Theory 60 (1996), no. 1, 23-41.

[3] M. Eie, A note on Bernoulli numbers and Shintani generalized Bernoulli polynomials, Trans. Amer. Math. Soc. 348 (1996), no. 3, 1117-1136.

[4] M.-S. Kim, A note on sums of products of Bernoulli numbers, Appl. Math. Lett. 24 (2011), no. $1,55-61$.

[5] M.-S. Kim and S. Hu, Sums of products of Apostol-Bernoulli numbers, Ramanujan J. 28 (2012), no. 1, 113-123.

[6] A. Petojević, New sums of products of Bernoulli numbers, Integral Transforms Spec. Funct. 19 (2008), no. 1-2, 105-114.

[7] H. M. Srivastava, Some formulas for the Bernoulli and Euler polynomials at rational arguments, Math. Proc. Cambridge Philos. Soc. 129 (2000), no. 1, 77-84.

[8] H. M. Srivastava and J. Choi, Series Associated with the Zeta and Related Functions, Kluwer Academic Publishers, Dordrecht, 2001. 
[9] H. M. Srivastava and Á. Pintér, Remarks on some relationships between the Bernoulli and Euler polynomials, Appl. Math. Lett. 17 (2004), no. 4, 375-380.

Department of Mechanical Engineering

KYUNGNAM UNIVERSITY

Changwon 631-701, Korea

E-mail address: sonjin@kyungnam.ac.kr 